\title{
Looking for the Evidence: Value of Health Informatics. Editorial
}

\author{
B. Séroussi ${ }^{1,2}$, M.-C. Jaulent ${ }^{3}$, C.U. Lehmann ${ }^{4}$ \\ 1 UPMC, UFR de Médecine, Sorbonne Universités, Paris, France; Hôpital Tenon, Département de Santé Publique, Paris, France \\ 2 Université Paris 13, Sorbonne Paris Cité, LIM\&BI0 (EA3969), Bobigny, France \\ ${ }^{3}$ INSERM, UMR S872, EQ20, Paris, France \\ ${ }^{4}$ Departments of Pediatrics and Biomedical Informatics, Vanderbilt University, Nashville, TN, USA
}

\begin{abstract}
Summary
Objectives: To provide an editorial introduction to the 2013 IMIA Yearbook of Medical Informatics with an overview of its contents and contributors.

Methods: A brief overview of the main theme, and an outline of the purposes, contents, format, and acknowledgment of contributions.

Results: Health information technology (HIT) is currently widely implemented to improve healthcare quality and patient safety while reducing costs. Although these benefits are expected and largely advertised, the evidence for these benefits is still missing. Unintended consequences are offen reported and some applications have been shown to be wasteful, harmful, and even fatal. Evidence-bosed health informatics has been defined as "the conscientious, explicit and judicious use of current best evidence when making decisions about the introduction and operation of information technology in a given health care setting". The 2013 issue of the IMIA Yearbook highlights important contributions about the significant challenges that arise from the assessment of HIT solutions. Progress towards evidence-bosed health informatics is identified to elicit what works, what doesn' $†$ work, and why. In an environment where resources are limited, budgets lower than in past years, and the need to improve care is becoming ever more pressing, focusing on this topic should guide institutions and providers in the implementation of the best health information technology.

Condusion: This overview of progress and current challenges across the spectrum of the discipline shows many great examples of evidence that have been gathered on the effectiveness of HIT. However, evidence remains limited and a significant work should be conducted to improve the development, testing, and implementation of HIT applications.
\end{abstract}

\section{Keywords}

Editorial, 2013 IMIA Yearbook of Medical Informatics, evidencebased health informatics, surveys of biomedical informatics, IMIA and its societies

Yearb Med Inform 2013:4-6

\section{Introducing the 2013 Yearbook and its Theme on Evidence- Based Health Informatics}

Health care worldwide has faced significant challenges - unsustainable inflation of costs, varying quality, obstacles to access, and challenges to patient safety. With the unprecedented implementation rate of electronic health records adoption, the increased use of personal health records, the proliferation of clinical decision support associated with ordering and documentation systems, it is easy to buy into the notion that health information technology (HIT) may be the panacea to all our health care problems. Research and reports from the field tend to support the notion - we have developed and implemented solutions that improve safety by preventing errors or identifying dangerous conditions, we have successfully shown cost reductions, improved education and access to care, and the ability to deliver higher quality of care.

However, if most HIT applications have been shown to be beneficial, some have proven to be wasteful, harmful, and even fatal. Unfortunately, inadequate applications are usually detected too late, after technology-induced errors are discovered and may have reached patients. We have became painfully aware that we lack knowledge to help us choose and select the best possible solutions from the glut of possible health information technology tools available to us, in order to address our problems in medicine. Clinicians know only too well that the availability of many different treatment options for a specific disease usually means that none of the options has proven or tested superior, leading to lack of standardization in care and variability in treatment with negative impact on patient outcomes. The same may be true for health information technology. How shall we select the best solutions from all offers? How shall we avoid failures by implementing a tool that has no safety benefits, increases cost, or is not accepted by users?

We chose Evidence-based Health Informatics as the theme for the 2013 Yearbook to draw attention to the work that is being done to identify what works and what doesn't work in health informatics. Our resources are limited, our budgets lower than in past years, and our need to improve care is becoming ever more pressing. Focusing on this topic will help to highlight successes and failures and will not only identify the direction of future research but more importantly the best choices institutions and providers can make to implement the best health information technology.

The first published use of the term evidence-based health informatics was in the mid-1990s, in the setting of McMaster University, from where the initial Evidence-Based Medicine Working Group was supported. Similar to Evidence-Based Medicine defined as "the conscientious, explicit, and judicious use of current best evidence in making decisions about the care of individual patients", evidence-based health informatics (EBHI) has been defined as "the conscientious, explicit and judicious use of current best evidence when making decisions about the introduction and operation of information technology in a given health care setting". The first cited absorption of this concept into the health informatics domain came at Medinfo in 2001, with recognition that "information systems are not different from any other health system, in needing to be evidence-based, and specified and implemented based on best evidence-based information". 
The core goals of EBHI are to ensure that policies and implementations are based on evidence (as opposed to the currently prevalent combination of aspiration and marketing promises); and to avoid iatrogenic or institutional damages caused by adverse selection or implementation of health informatics applications. Achievement of this requires the development and use of scientific approaches to obtain the evidence. As indicated below a number of advocates have been pursuing this vision, which has then been facilitated by concerted action between the IMIA and EFMI Working Groups covering this responsibility.

The ongoing drive to implement HIT however opens the door to unlimited opportunities to test, evaluate, and compare solutions and their impact on care. We are collecting unprecedented amounts of data such as orders, physician and nurse documentation, medication administration, laboratory and radiological data and interpretation, self reported symptoms, diet and exercise behavior and much more. The ability to extract and analyze these data in the context of clinical decision support solutions ushers in the new epoch of evidence generation and with it the ability to make the best possible choices to select the best clinical decision support and information technology solution. Until now, the evaluation of computer decision support solutions has focused on process measures such as "Have we given the immunization?" or "Has the patient received a pamphlet in her language?" What health care needs are fewer process measures and more patient or population outcome-based measures tying health information technology to evidence of its effectiveness, cost, and possible side effects.

One of the benefits of being the editors of a Yearbook is the opportunity to focus on a specific topic that in the editors' opinions deserves more attention. However, more importantly, editors are allowed to invite and publish thoughts provoking and sometimes controversial manuscripts. Dr. Koppel's keynote is such a thought provoking piece. About the evidence of HIT, Dr. Koppel keeps reminding us that the emperor is not wearing any clothes - that evidence is still too limited and patchy, and he chides us for being too eager to leap onto the assumptions that technology will be cure for everything. He also challenges us to do more: to create more transparency, publish results of evaluations, create standards, most importantly remain skeptical and independent in our thinking. On the contrary, survey papers attempted to emphasize evidence that HIT works. However, when conducting literature searches to collect quality paper to evaluate HIT impact (randomized controlled trials or study designs with high strength of evidence), they generally found very few of them and none in developing countries, leading to the conclusion that "more evaluations are needed to demonstrate evidence".

In conclusion, evidence of the benefits of HIT in the health and wellness of patients remains limited. As the keynote points out, evidence is not only lacking but we are seeing increasing evidence that poor design, implementation, or workflow may cause harm to our patients. If this Yearbook shows many great examples of evidence that has been gathered on the effectiveness of HIT, it alerts us to the fact that significant work and research should be conducted to improve the development, testing, and implementation of HIT applications as well as end user training. We would be remiss if we were not to call on our readers to join the effort of creating, gathering, and curating more evidence. Our patients and providers deserve more usability testing and evaluation, post-deployment monitoring and patient safety evaluations. We, the editors, are looking forward to include your contributions to a future Yearbook. It is your work that will drive us closer to our goal. The road to evidence-based health information technology is long and arduous and we most certainly will encounter many failures and detours. But the goal is worth the travel - better care through knowledge.

The 2013 IMIA Yearbook includes the following articles:

Keynote on the theme of Evidence-Based Informatics - Ross Koppel

Editorial, IMIA President Statement, Synopses and summaries of Best Paper Selections in each of the subfields of biomedical informatics.

As in previous editions of the Yearbook, we could find the section dedicated to the main theme or "Special Session", as well as usual sections on Health and Clinical Management (HCM), Human Factors (HF), Health Information Systems (HIS), Sensor, Signal and Imaging Informatics (SSII), Decision Support (DS), Knowledge Representation and Management
(KRM), Education and Consumer Health Informatics (ECHI) and Bioinformatics (BTI).

We are very pleased to announce the introduction, in 2013, of a new section, the Clinical Research Informatics (CRI) section. Since 2009, CRI has emerged as a distinct sub-discipline of informatics that faces an explosive growth of clinical research data and information as well as an increasingly complex workflow and information management challenges. Current research in this field includes specific topics such as - optimal protocol design and efficient management; - patient recruitment and management; - adverse event reporting or regulatory compliance. This new field attempts to share and reuse data from electronic healthcare records and interoperable information systems to lower the barriers to clinical and translational research and to accelerate the translation of research into clinical practice.

Surveys authored in selected subfields of health informatics include (only the first author is named):

- Allison B. McCoy (Special section): State of the Art in Clinical Informatics - Evidence and Examples

- Patricia Abbott (HCM): A Scoping Review of Telehealth

- Marie-Catherine Beuscart-Zephir (HF): Evolution of Human Factors Research and Studies of Health Information Technologies: The Role of Patient Safety

- Dipak Kalra (HIS): A Review of the Empirical Evidence of the Healthcare Benefits of Personal Health Records

- Richard Shiffman(DS): Evidence-Based Clinical Decision Support

- Stefan Schulz (KRM): Recommendations for Formal Ontologies in Biomedical Knowledge Representation

- Michael C. Gibbons (CI): Personal Health and Consumer Informatics - The Impact of Health Oriented Social Media Applications on Health Outcomes

- Indra N. Sarkar (BTI): Bringing Genome Tests into Clinical Practice

- Peter J. Embi (CRI): Clinical Research Informatics: Survey of Recent Advances and Trends in a Maturing Field

IMIA working group papers:

- Elizabeth M. Borycki: Usability Methods for Ensuring Health Information System Safety: Evidence-Based Approaches 
- Daniel Luna: Health Informatics in Developing Countries: Systematic Review of Reviews

- Michael Rigby: Evidence Based Health Informatics -10 Years of Efforts to Promote the Principle

- Andre Kushniruk: From Usability Testing to Clinical Simulations: Bringing Context into the Design and Evaluation of Usable and Safe Health Information Technologies

- Holger Schmuhl: Utilization of Open Source Software in German and European Health Care Delivery - Results of a Qualitative Field Study

- Harshana Liyanage: The Evidence-base for Using Ontologies, Semantic Integration Methodologies to Support the Integrated Management of Chronic Disease in Primary and Ambulatory Care: Realist Review

- Talya Miron-Shatz: Social Media for the Promotion of Holistic Self-Participatory Care: An Evidence Based Approach

Research \& Education:

- Omar Bouhaddou: Medical Informatics in Morocco

History of Medical Informatics:

- Mikko Korpela: Two Decades of HELINA Conferences: A Historical Review of Health Informatics in Africa

- Jana Zvarova: Medical Decision Support and Medical Informatics Education: Roots, Methods and Applications in Czechoslovakia and the Czech Republic

\section{About the Yearbook of Medical Informatics}

The Yearbook of Medical Informatics of the International Medical Informatics Association (IMIA) is distributed through IMIA's Member and Corresponding Member Societies worldwide. Since its inception in 1992 it has been the single most important publication summarizing the activities of IMIA, and showcasing the best of medical informatics research for the previous year. Its original survey articles give overviews of recent developments. The Yearbook remains a non-profit publication of IMIA, jointly published with Schattauer Verlag and indexed in PubMed. It is currently subscribed to by 23 of IMIA's member societies, providing access for about 25.000 individual members.

\section{Information on IMIA and on its Regional Groups}

The Yearbook contains detailed information about IMIA, its Member Societies, Regional Groups, Working Groups, and Special Interest Groups. A report on the activities of two IMIA regions is included with the help of Regional Editors. We would like to thank Elisabeth Borycki, Don Newsham, and David Bates (for the North American IMIA Member Societies) for their original contribution on the current status of electronic health record (EHR) adoption and implementation in Canada and the United States and A. Moen and al. (for EFMI) for their valuable contribution on the status and challenge of eHealth in Europe.

\section{Acknowledgements}

The editors gratefully acknowledge the contributions of the referees and guest editors. They would also like to thank the authors of the invited surveys, reviews, and the historical paper, and the contributors to the Research and Education Section. They are most appreciative of the considerable skill, time, and effort devoted by the Section Editors, Christian Lovis, Nathalie Souf, Xavier Aimé, Gunnar Declerck, Sandrine Voros, Alexandre Moreau-Gaudry, Jacques Bouaud, Jean-Baptiste Lamy, Jean Charlet, Stefan Darmoni, Nicolas Griffon, Pascal Staccini, Nassim Douali, Thierry Lecroq, Lina Soualmia, Christel Daniel, Remy Choquet. They especially wish to thank the Editorial Assistant, Martina Hutter, from the Medical Faculty at the University of Heidelberg, without whose untiring efforts the Yearbook would not have been completed.

The editors appreciate the contributions of the Advisory Board to the planning of this Yearbook. They are Reinhold Haux, Victor Maojo and George Mihalas.

The referees who contributed to the selection of articles in the 2013 Yearbook of Medical Informatics were:

Ameen Abu-Hanna, The Netherlands Samantha A. Adams, The Netherlands

Kouhei Akazawa, Japan

Najeeb Al-Shorbaji, Switzerland

Manfredo Atzori, Switzerland
Samy A. Azer, Saudi Arabia

Cheikh Oumar Bagayoko, Mali

Thomas Beale, Australia

Mohammed Bennani, Morocco

Fernan Gonzalez Bernaldo de Quiros, Argentina

Elmer Bernstam, USA

Régis Beuscart, France

Olivier Bodenreider, USA

Omar Bouhaddou, USA

Alex A.T. Bui, USA

Anita Burgun-Parenthoine, France

Jean Charlet, France

James C. Cimino, USA

Ronald Cornet, The Netherlands

Karen Courtney, Canada

Thierry Dart, France

Guilherme Del Fiol, USA

John T. Donnelly, USA

Catherine Duclos, France

Georg Duftschmid, Austria

Martin Dugas, Germany

Jacques Felblinger, France

Dragan Gamberger, Croatia

Thomas Ganslandt, Germany

Natalia Grabar, France

Adela Grando, United Kingdom

Ragnhild Helleso, Norway

Carmen Hernández Rabanal, Spain

Michael Hogarth, USA

Andreas Holzinger, Austria

Dimitar Hristovski, Slovenia

Josef Ingenerf, Germany

Clement Jonquet, France

Jayashree Kalpathy-Cramer, USA

Hong-Gee Kim, Korea

Richard Lenz, Germany

Giorgio Leonardi, Italy

Yves A. Lussier, USA

Farah Magrabi, Australia

Philippe Massonet, Belgium

Maryati Mohd Yusof, Malaysia

Fleur Mougin, France

Jeannette Murphy, United Kingdom

Toshiharu Nakai, Japan

Else Nygren, Sweden

Christian Ohmann, Germany

Vimla Patel, USA

Sylvia Pelayo, France

Natalia Pletevna, Switzerland

Michael Poprat, Germany

Rebecca Randell, United Kingdom

Rainer Röhrig, Germany

Valeri Sintchenko, Australia

Tassos Tagaris, Greece

Guy Tsafnat, Australia

Frank Ückert, Germany

Alfonso Valencia, Spain

Kai Zheng, USA

Xue Zhong Zhou, USA

Tatjana Zrimec, Australia 